\title{
Scholarly Research Infrastructures Stakeholder Group Report
}

\author{
Lars Bjornshange
}

\section{Definition}

Infrastructure /'infrə, $\operatorname{str} \mathrm{k}(\mathrm{t}) \mathrm{SH} \mathrm{H}$ /

(noun)

The basic physical and organizational structures and facilities (e.g. buildings, roads, power supplies) needed for the operation of a society or enterprise. New Oxford American Dictionary

\section{Infrastructures in the context of Open Scholarly Communication}

Standardized communication protocols, data exchange formats, metadata standards, harvesting protocols, APIs, identifiers (DOI, authorID (ORCID), organizational ID, funderIDs) and facilitating services. This list is far from complete.

\section{Background}

The stakeholder group agrees that

- Infrastructures, standards etc. are crucial for making open possible

- That the drivers for infrastructures, standards, identifiers and other bits and pieces of infrastructure in scholarly communication have (and still are) originated from the North/West

- That new bits and pieces of infrastructures need to be developed. For example, bits and pieces to facilitate handling and monitoring of APC-payments

\section{Major issues}

- Global implementation/adoption of infrastructures

- Governance and sustainability of open infrastructures to support Open Scholarship

\section{Global implementation / adoption}

In so far as OSI aspires to facilitate a Global Open Scholarly Communication System, we must realize that

- Much of the bits and pieces of infrastructure have been developed without sufficient consultation with the global community

- This has serious implications for the implementation/adoption of infrastructure

(C) 2017 OSI2017 Scholarly Research Infrastructures Stakeholder group. This open access article is distributed under the Creative Commons Attribution 4.0 International License. This document reflects the combined input of the authors listed here (in alphabetical order by last name) as well as contributions from other OSI2017 delegates. The findings and recommendations expressed herein do not necessarily reflect the opinions of the individual authors listed here, nor their agencies, trustees, officers, or staff. 
This in turn means that today we cannot talk about a Global Open Scholarly Communication System There are serious barriers to implementation, including: lan-

guages, geography, cultures

\section{Governance and sustainability of Open Infrastructures to support Open Scholarship}

Given that research transcends disciplines, geography, institutions and stakeholders, the infrastructure that supports it needs to do the same. ${ }^{1}$

Infrastructures should

- Be stakeholder governed

- Have transparent operations

- Have sufficient resources to be available to secure global adoption

\section{What OSI should do}

- scan the current bits and pieces of infrastructure and evaluate their adoption on a global scale

- engage with the "owners" of the infrastructures to push for measures that can secure global implementation/adoption

If infrastructures are not implemented/ adopted on a global scale we cannot talk about a Global Open Scholarly Communication System and the flow of research outputs (papers, data and software) on a global scale will continue to be broken!

\section{Notes}

1. Inspiration for this discussion can be found here: Bilder G, Lin J, Neylon C (2015). Principles for Open Scholarly Infrastructure-v1. Retrieved July 9, 2017 http://dx.doi.org/10.6084/m9.figshare.1314859

\section{Further Reading}

Work in progress on the sustainability of Open Infrastructures:

- Knowledge Exchange (http://www.knowledge-exchange.info/) has done a lot of work on this. See: Sustainability of Open Access Services; (Retrieved July 9, 2017 : http://www.knowledge-exchange.info/event/sustainability-oa-services). See also "Putting down roots: Securing the future of Open Access policies," January 2016 (Retrieved July 9, 2017: http:// repository.jisc.ac.uk/6269/10/final-KE-Report-V5.120JAN2016.pdf).

- Continuation of the work is facilitated by SPARC Europe. (Retrieved July 9, 2017: http://sparceurope.org/global-sustainability-coalition-open-science-servicesestablished/) 